\title{
Erratum to: In vivo molecular profiling of human glioma using diffusion kurtosis imaging
}

\author{
Johann-Martin Hempel ${ }^{1}$ - Sotirios Bisdas ${ }^{1,2}$ - Jens Schittenhelm ${ }^{3}$. \\ Cornelia Brendle $^{1} \cdot$ Benjamin Bender $^{1} \cdot$ Henk Wassmann ${ }^{1} \cdot$ Marco Skardelly $^{4}$. \\ Ghazaleh Tabatabai $^{5} \cdot$ Salvador Castaneda Vega $^{6} \cdot$ Ulrike Ernemann $^{1} \cdot$ Uwe Klose $^{1}$
}

Published online: 22 September 2016

(C) Springer Science+Business Media New York 2016

\section{Erratum to: J Neurooncol \\ DOI 10.1007/s11060-016-2272-0}

In the initial online publication, the second author's family name was incorrect. The name has been updated in the online article and is correct in this erratum too.

The online version of the original article can be found under doi:10.1007/s11060-016-2272-0.

Johann-Martin Hempel

johann-martin.hempel@uni-tuebingen.de

1 Department of Neuroradiology, Eberhard Karls University, Tübingen, Germany

2 Department of Neuroradiology, National Hospital of Neurology and Neurosurgery, University College London Hospitals, London, UK

3 Institute of Neuropathology, Eberhard Karls University, Tübingen, Germany

4 Department of Neurosurgery, Eberhard Karls University, Tübingen, Germany

5 Centre of Neurooncology, Comprehensive Cancer Center Tübingen-Stuttgart, Eberhard Karls University, Tübingen, Germany

6 Department of Preclinical Imaging and Radiopharmacy, Werner Siemens Imaging Center, Eberhard Karls University, Tübingen, Germany 